\title{
An Efficient Approach toward Color Image Segmentation with Combined Effort of Soft Clustering and Region Based Techniques using L Channel of LAB Color Space
}

\author{
Dibya Jyoti Bora \\ Department of Computer \\ Science \& Applications \\ Barkatullah University \\ Bhopal
}

\author{
Anil Kumar Gupta \\ Department of Computer \\ Science \& Applications \\ Barkatullah University \\ Bhopal
}

\author{
Fayaz Ahmad Khan \\ Department of Computer \\ Science \& Applications \\ Barkatullah University \\ Bhopal
}

\begin{abstract}
Finding an efficient approach for color image segmentation is always sought by the researchers in the color image processing research. We have different clustering based and region based methods for the same. But still there arises the requirement of an optimal method. In this paper, a new approach for color image segmentation is proposed. Here the segmentation is carried out on the $\mathrm{L}$ channel of LAB color space. The input color image is first converted from RGB to $\mathrm{LAB}$. Then $\mathrm{L}$ channel is extracted from the LAB converted image and sent as input to FCM algorithm. After this initial segmentation, the segmented image is filtered by sobel filter. The filtered image is then segmented by Meyer's Watershed algorithm to produce the final segmented image of the original image. The results of the proposed approach are found efficient when the same are analyzed in terms of MSE and PSNR. Also the segmented images are found free from over segmentation.
\end{abstract}

\section{General Terms}

Image Processing

\section{Keywords}

Color Image Segmentation, FCM, LAB, Sobel Filter and Watershed Algorithm

\section{INTRODUCTION}

In image processing research, image segmentation always plays a crucial rule. It is the most important phase as the result of which, the whole image analysis process depends. Image segmentation can be defined as a process of assigning a label to every pixel in an image such that pixels with the same label share certain visual characteristics. As a result of image segmentation process, the image is divided into a set of segments which are homogeneous with respect to some criteria like color, intensity, or texture [1]. Let I is the given image. As a result of image segmentation, it will be partitioned into ' $n$ ' disjoint partitions $\mathrm{R}_{\mathrm{i}}(\mathrm{i}=1,2, . ., \mathrm{n})$ so that the following properties will be satisfied[2][3]:
(i) $\bigcup_{i=1}^{n} R_{i}=R$
(ii) $\quad R_{i} \bigcap R_{j}=\Phi$

(iii) $\mathrm{H}\left(\mathrm{R}_{\mathrm{i}}\right)=$ TRUE $\forall \mathrm{i}$

(iv) $\mathrm{H}\left(\mathrm{R}_{\mathrm{i}} \cup \mathrm{R}_{\mathrm{j}}\right)=$ FALSE $\forall \mathrm{R}_{\mathrm{i}} \& \mathrm{R}_{\mathrm{j}}$ adjacent.

Here, $H(R)$ denotes the homogeneity attributes of pixels over region $\mathrm{R}$ on the basis of which the whole segmentation process carried out [3]. So, it is obvious from (iii) that pixels within a cluster must share same featured components. And the property (iv) implies that if pixels belong to two different clusters then their featured components must also be different from each other. Image segmentation may be gray or color. Color image segmentation is sometimes found more beneficial than gray scale segmentation because of the reason that human eyes are more adjustable to brightness, so, can identify thousands of color at any point of a complex image, while only a dozens of gray scale are possible to be identified at the same time [4].There exist different methodologies for color image segmentation process among which clustering is frequently chosen one [5]. Also region based methods show good performance in color image segmentation. Our proposed approach is a combination of both clustering and region based techniques. Here FCM, one of the famous soft clustering techniques is used for initial segmentation of the image. And for final segmentation, Mayer's watershed algorithm is chosen. The result of the color segmentation depends on choosing a proper color space [6] and it is seem that HSV and $\mathrm{LAB}$ are two frequently chosen color space for this purpose. Here, we have chosen LAB color space. The L Channel of the $\mathrm{LAB}$ converted image is sent as input to the FCM algorithm. The FCM segmented image is then filtered with sobel filter and Mayer's Watershed algorithm is applied on the filtered image. Thereby, the resultant segmented image is obtained.

The rest of the paper is organized as follows: Section 2 gives a brief review on the work done in the field. The flowchart of the proposed approach is presented in the Section 3. Then the topics involved in the proposed approach : LAB color space, FCM algorithm, Sobel Filter And Watershed algorithm are discussed in the sections 4, 5,6 and 7 respectively. Experiment and results are presented in the section 8 . Finally, conclusion and future scope is given in the section 9.

\section{REVIEW OF LITERATURES}

In [7], the authors proposed an approach where they combine segmentation of various color spaces such as RGB, HSV, YIQ and $\mathrm{XYZ}$ to give more accurate segmentation result compared to segmentation of single color space. K-Means and Effective robust kernelized fuzzy c-means (ERKFCM) are used to 
segment the images. The performance is evaluated using MSE and PSNR.

In [8], an image segmentation method is proposed where the use of fuzzy c-means clustering in image segmentation is shown. This method is based on a basic region growing method and uses membership grades' of pixels to classify pixels into appropriate segments. Images were in RGB color space, as feature space was used $\mathrm{L}^{*} \mathrm{u}^{*} \mathrm{v} *$ color space. Results of the experiments showed that the method is very efficient in dealing with color image segmentation but one of the demands was not reached that was the demand of simplifying borders.

In [9], an optimized fuzzy logic method for Magnetic Resonance Imaging (MRI) brain images segmentation is presented. This technique is based on a modified fuzzy cmeans (FCM) clustering algorithm. The FCM algorithm that incorporates spatial information into the membership function is used for clustering. The advantages of the algorithm are that it is less sensitive to noise than other techniques, and it yields regions more homogeneous than those of other methods.

In [10], the authors proposed a new fuzzy c means algorithm that relies on a new efficient cluster center initialization and color quantization allowing faster and more accurate convergence such that it is suitable to segment very large color images. The experiments on real images proved the efficiency of the algorithm in terms of both accuracy and computation time.

In [11], a novel approach for clustering based image segmentation is used. Here, RGB image is first converted to $\mathrm{LAB}$ one. The LAB image is then segmented with $\mathrm{K}$ Means algorithm with Cosine distance measure. The segmented image is then filtered with sobel filter and sent as input to the Watershed algorithm to obtain the final segmentation. The results are found quite satisfactory in terms MSE and PSNR values.

In [12], a new method for image segmentation based on watershed transformation using mathematical morphology is proposed. To avoid over segmentation, the topological gradient method is adopted. The results obtained are found very efficient.

In [13], a modified version of watershed algorithm is presented where an adaptive masking and a threshold mechanism are used over each color channel to overcome the over segmentation problem of watershed algorithm, before combining the segmentation from each channel to the final one. The approach is enhancing the segmentation result and also result is found more accurate as per the obtained values of image quality assessment metrics such as PSNR, MSE, PSNRRGB and Color Image Quality Measure (CQM) based on reversible YUV color transformation.

In [14], the two most commonly used edge detection methods (Laplacian and Sobel edge detectors) are discussed. The Sobel edge detection algorithm is found performing better than Laplacian algorithms, but the false edges are high in both cases for blurred or low resolution images. To solve this problem,the authors proposed a new algorithm and set of filters (kernels) similar to sobel method. The proposed method performs a 2-D spatial gradient quantity on an image and highlight regions of high spatial frequency corresponding to edges. The kernels are designed to respond maximally to edges running diagonally in addition to vertically and horizontally relative to the pixel grid, one kernel for each of the two perpendicular orientations. The proposed operator consists of a pair of $3 \times 3$ convolution kernels. From the results obtained it is found that the proposed algorithm performs better than in terms of less false edges than the Sobel and Laplacian filters.

\section{FLOWCHART OF THE PROPOSED APPROACH}

The steps involved in the proposed approach can be best understood by the following flowchart:

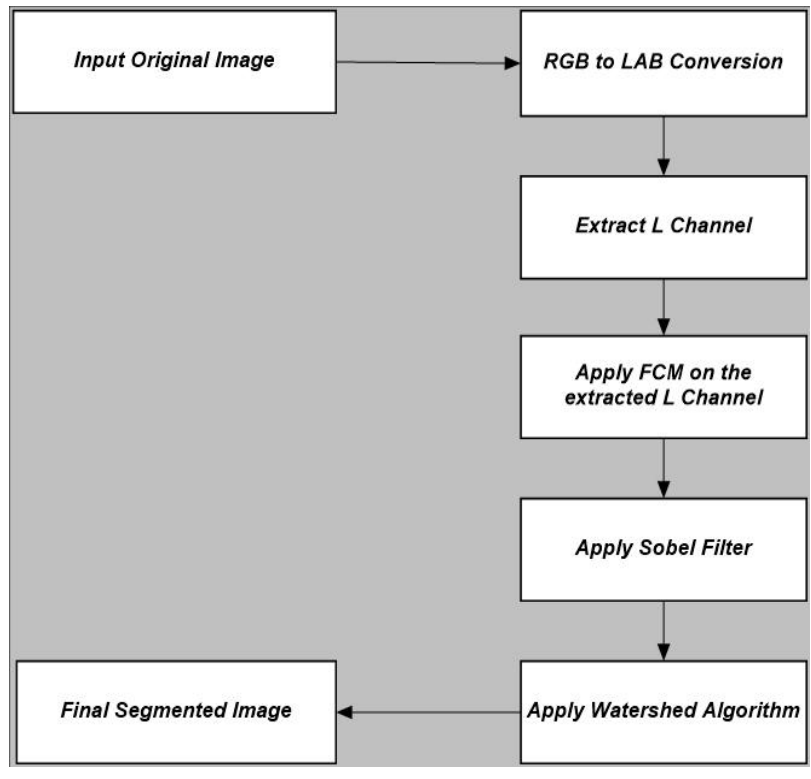

Fig1: Proposed Approach

\section{LAB COLOR SPACE}

The $\mathrm{LAB}$ color space $(\mathrm{L} * \mathrm{~A} * \mathrm{~B} *)$ is defined by $\mathrm{CAE}$ and specified by the International Commission on Illumination [15][16]. Here L is for Luminance (Lightness) and other two color channels are $\mathrm{a}$ and $\mathrm{b}$ known as chromaticity layers. The $a^{*}$ layer indicates where the color falls along the red green axis, and $b^{*}$ layer indicates where the color falls along the blue-yellow axis. $a^{*}$ negative values indicate green while positive values indicate magenta; and $b^{*}$ negative values indicate blue and positive values indicate yellow. The device independency property of this color space makes it a very unique and useful one as this property provides us the opportunity to communicate different colors across different devices. Let's understand this color space more clearly with the following figure [17]:

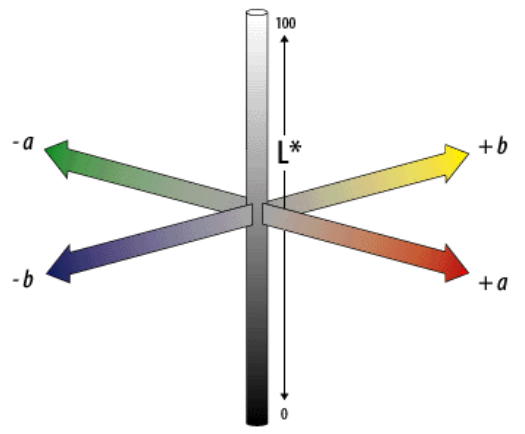

Fig 2: $\mathbf{L} * \mathbf{A} * \mathbf{B} *$ Color Space

In the above figure, the central vertical axis represents lightness (L*). $\mathrm{L}^{*}$ can take values from 0 (black) to 100 (white). The coordinate axes follows the fact that a color 
cannot be both red or green, or both blue and yellow, because these colors oppose each other. Values run from positive to negative for each axis. Positive 'a' values indicate amounts of red, while negative values indicate amounts of green. And, positive ' $b$ ' values indicate amount of yellow while, negative 'b' values indicate blue. The zero represents neutral gray for both the axes. So, here, values are needed only for two color axes and for the lightness or grayscale axis $\left(\mathrm{L}^{*}\right)$.

\section{FCM ALGORITHM}

Among the available soft clustering algorithms, FCM (Fuzzy C Means) is the most frequently chosen one. Here, each data point belongs to a cluster to some degree that is specified by a membership grade [18]. This membership grades(which are values in $[0,1])$ gives the flexibility to express that data points can belong to more than one cluster. The algorithm iteratively tries to minimize the cost function:

$$
J_{m}=\sum_{i=1}^{N} \sum_{j=1}^{C} u_{i j}{ }^{m}\left\|x_{i}-c_{j}\right\|^{2}
$$

In the above equation, $\mathrm{m}$ is any real number greater than $1, u_{i j}$ is the degree of membership of pixel $\boldsymbol{x}_{i}$ in the cluster $j,\|*\|$ is any norm expressing the similarity between the concerned pixel and the center, the parameter $m$ controls the fuzziness of the partition[19]. Fuzzy partitioning is carried out through an iterative optimization of the objective function shown above, with the update of membership $u_{i j}$ and the cluster centers $c j$ by

$$
\begin{aligned}
& u_{i j}= \frac{1}{\sum_{k=1}^{c}\left(\frac{\left\|x_{i}-c_{j}\right\|}{\left\|x_{i}-c_{k}\right\|}\right)^{\frac{2}{m-1}}} \\
& c_{j}=\frac{\sum_{i=1}^{N} u_{i j}{ }^{m} \bullet x_{i}}{\sum_{i=1}^{N}{u_{i j}{ }^{m}}^{m}}
\end{aligned}
$$

The iteration will stop if $\max _{i j}\left\{\left|u_{i j}{ }^{(k+1)}-u_{i j}{ }^{(k)}\right|\right\}<\in$ where $\in$ is a termination criteria between 0 and 1 , whereas $\mathrm{k}$ are the iteration steps. This procedure converges to a local minimum or a saddle point of $J_{m}$. In our proposed approach, the extracted $\mathrm{L}$ channel of the LAB converted image is undergone an initial segmentation by FCM.

\section{SOBEL FILTER}

Sobel filter (also, known as Sobel operator), named after Irwin Sobel [20], is an edge detection algorithm. This is generally used to create an image by emphasizing edges and transitions of the image. Sobel filter is a discrete differentiation operator which computes an approximation of the gradient of the image intensity function. This computation is based on convolving the image with a small, separable, and integer valued filter in horizontal and vertical direction and is therefore relatively inexpensive in terms of computations [21]. Sobel filter is an orthogonal gradient operator, where gradient corresponds to first derivative and gradient operator is a derivative operator [22]. Two kernels: Gx and Gy are involved for every image ; where Gx is estimating the gradient in $\mathrm{x}$-direction while Gy estimating the gradient in $\mathrm{y}$ direction. Then the absolute gradient magnitude will be given by:
$|\mathbf{G}|=\sqrt{ }\left(\mathbf{G x}^{2}+\mathbf{G} \mathbf{y}^{2}\right)$

Although, this value is often approximated with [21][22] :

$|\mathbf{G}|=|\mathbf{G x}|+|\mathbf{G y}|$

We have chosen sobel operator because of its capacity of smoothing effect on the random noises of an image [22]. The edge elements, being differentially separated by two rows and columns on both sides, become enhanced which offer a very bright and thick look of the edges.

\section{WATERSHED TRANSFORMATIONS}

Watershed transformation can be classified as a region-based segmentation approach [23]. The main concept of this algorithm is derived from geography. In geography, the term "watershed", means the ridge that divides areas drained by different river systems. When an image is viewed as geological landscape, the watershed lines determine boundaries which separate image regions. The watershed transform computes catchment basins and ridgelines (also known as watershed lines), where catchment basins corresponding to image regions and ridgelines relating to region boundaries. A diagrammatic view of watershed lines and catchment basins is [24]:

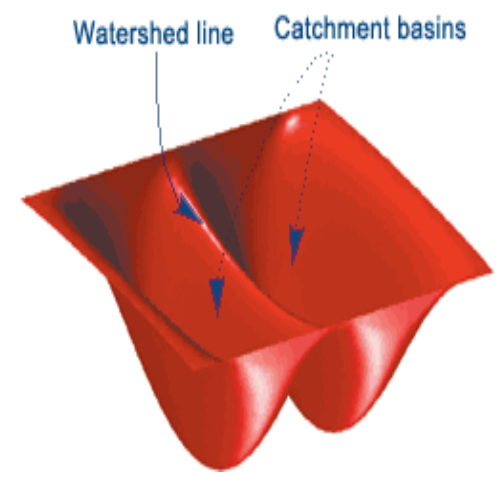

Fig 3: Watershed line and Catchment basins

For our proposed approach, Meyer's watershed algorithm is used for the final segmentation stage. Meyer's watershed algorithm consists of the following basic steps [25]:

1. Add neighbors to priority queue, sorted by value.

2. Choose local minima as region seeds.

3. Take top priority pixel from queue

1. If all labeled neighbors have same label, assign to pixel

2. Add all non-marked neighbors

4. Repeat step 3 until finished.

\section{EXPERIMENTS \& RESULTS}

We have chosen Matlab for the implementation of our proposed approach. The images for the experiments are collected from Matlab demo images [26] and Berkeley Image Segmentation dataset [27]. Following two measures are used to find the accuracy of the segmentation result:

The MSE (Mean Squared Error)is the cumulative squared error between the segmented image and the original image, whereas PSNR(Peak Signal to Noise Ratio) is the peak error[28]. The formula for MSE[28] is:

$\operatorname{MSE}=\sum_{y=1}^{M} \sum_{x=1}^{N}\left[I(x, y)-I^{\prime}(x, y)\right]^{2}$ 
where, $I(x, y)$ is the original image, $I^{\prime}(x, y)$ is its noisy approximated version (which is actually the segmented image) and $\mathrm{M}, \mathrm{N}$ are the dimensions of the images value for MSE implies lesser error.

The formula for PSNR[28] is:

$\mathrm{PSNR}=10 \log _{10}\left(M A X i^{2} / M S E\right)$

Where, MAXi is the maximum possible pixel value of the image. A higher value of PSNR is always preferred as it implies the ratio of Signal to Noise will be higher. 'signal' here is the original image, and the 'noise' is the error in reconstruction.

Experiment for Image 1:

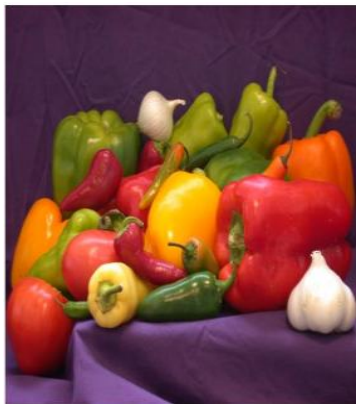

Fig 4(i): Original Image (Image 1)

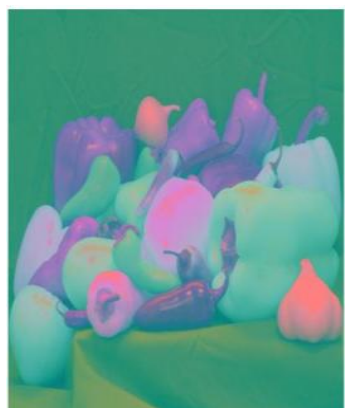

Fig 4(ii): LAB Converted Image

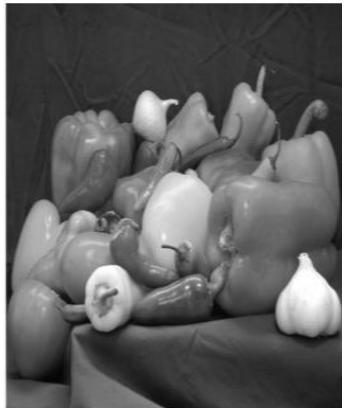

Fig 4(iii): L Channel

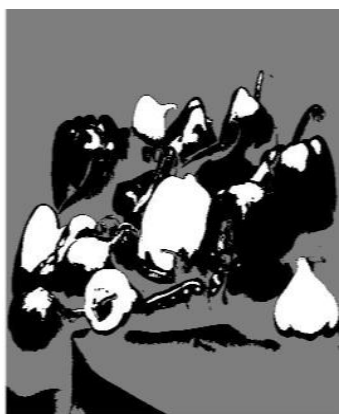

Fig 4(iv): FCM Segmentation

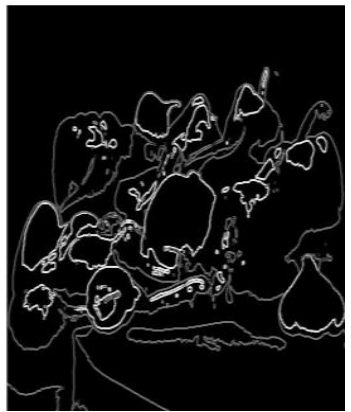

Fig 4(v): After Applying Sobel Filter

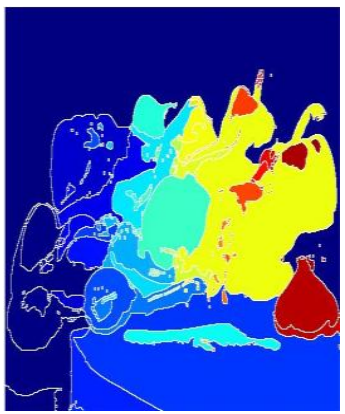

Fig. 4(v): Final Segmentation by Watershed Algorithm

Table 1.1: MSE \& PSNR values for Proposed Approach

\begin{tabular}{|l|l|}
\hline MSE & PSNR \\
\hline MSE(:,:,1) $=2.4114 \mathrm{e}+03$ & PSNR(:,:,1) $=14.3082$ \\
\hline MSE(:,:,2) $=2.2762 \mathrm{e}+03$ & PSNR(:,:,2) $=14.5587$ \\
\hline MSE(:,:,3) $=3.4920 \mathrm{e}+03$ & PSNR(::,:3) $=12.7000$ \\
\hline
\end{tabular}

Table 1.2: MSE \& PSNR values for Watershed Approach

\begin{tabular}{|l|l|}
\hline MSE & PSNR \\
\hline MSE(:,:,1) $=3.0102 \mathrm{e}+03$ & $\operatorname{PSNR}(:,:, 1)=13.3449$ \\
\hline $\operatorname{MSE}(:,:, 2)=5.0355 \mathrm{e}+03$ & $\operatorname{PSNR}(:,:, 2)=11.1104$ \\
\hline $\operatorname{MSE}(:,:, 3)=4.3078 \mathrm{e}+03$ & $\operatorname{PSNR}(:,:, 3)=11.7883$ \\
\hline
\end{tabular}

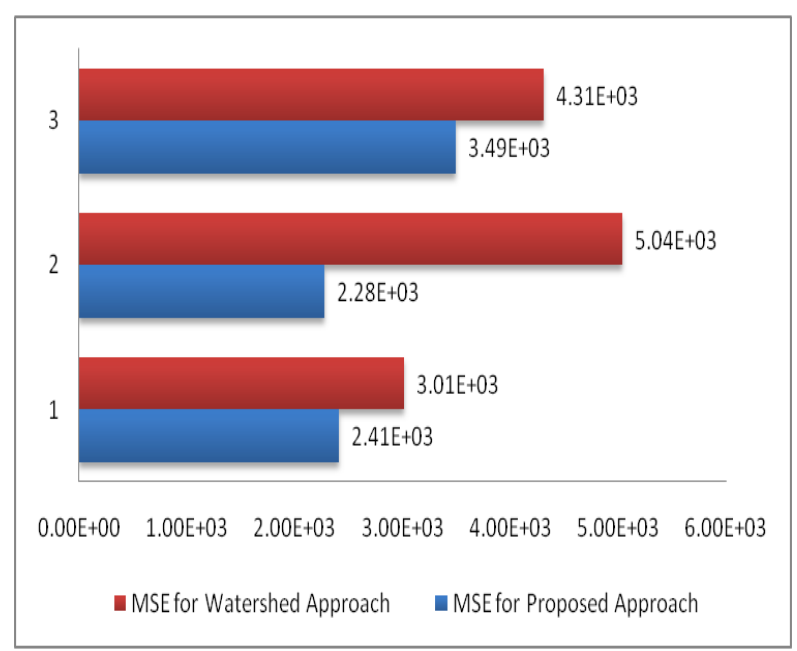

Chart 1.1: MSE Comparison for the Image 1 


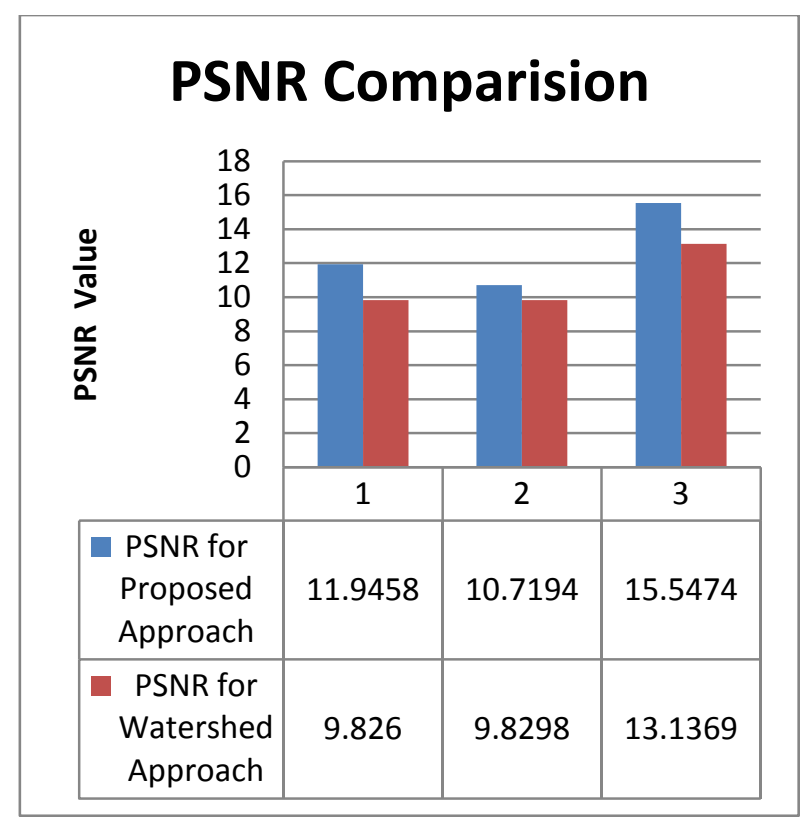

Chart 1.2: PSNR Comparison for the Image 1

Experiment for Image 2

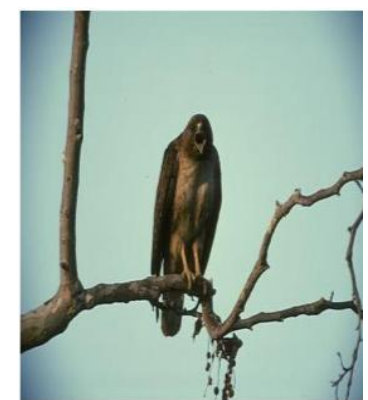

Fig 5(i): Original Image (Image 2)

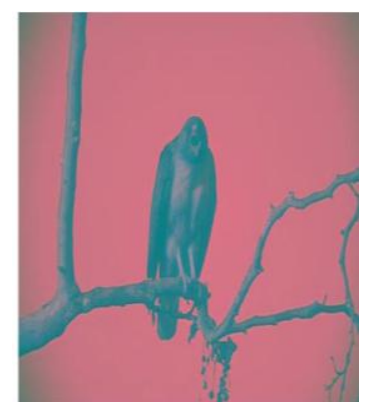

Fig.5 (ii): LAB Converted Image

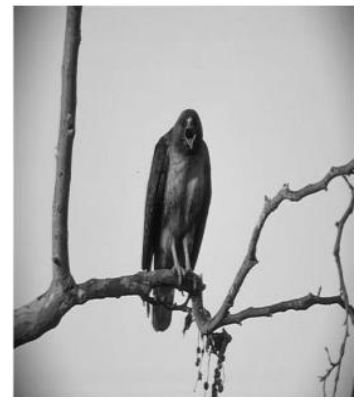

Fig 5(iii): L Channel

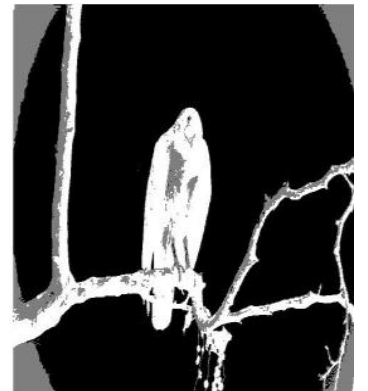

Fig 5 (iv): FCM Segmentation

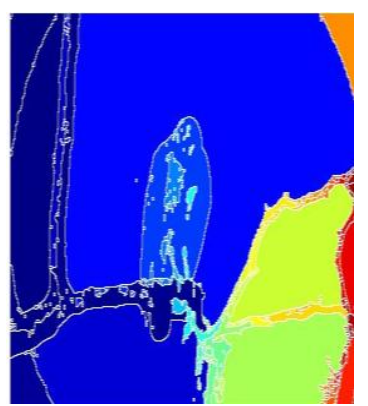

Fig 5(v): Final Segmentation by Watershed Algorithm

Table 2.1: MSE \& PSNR values for Proposed Approach

\begin{tabular}{|l|l|}
\hline MSE & PSNR \\
\hline MSE(:,:,1) $=4.1543 \mathrm{e}+03$ & $\operatorname{PSNR}(:,:, 1)=11.9458$ \\
\hline $\operatorname{MSE}(:,:, 2)=5.5099 \mathrm{e}+03$ & $\operatorname{PSNR}(:, ;, 2)=10.7194$ \\
\hline MSE $(:,:, 3)=1.8128 \mathrm{e}+03$ & $\operatorname{PSNR}(:,:, 3)=15.5474$ \\
\hline
\end{tabular}

Table 2.2: MSE \& PSNR values for Watershed Approach

\begin{tabular}{|l|l|}
\hline MSE & PSNR \\
\hline MSE(:,:,1) $=6.7683 \mathrm{e}+03$ & PSNR(:,:,1) $=9.8260$ \\
\hline MSE(:,:,2) $=6.7624 \mathrm{e}+03$ & PSNR(:,:,2) $=9.8298$ \\
\hline MSE(:,:,3) $=3.1579 \mathrm{e}+03$ & PSNR(::,:3) $=13.1369$ \\
\hline
\end{tabular}

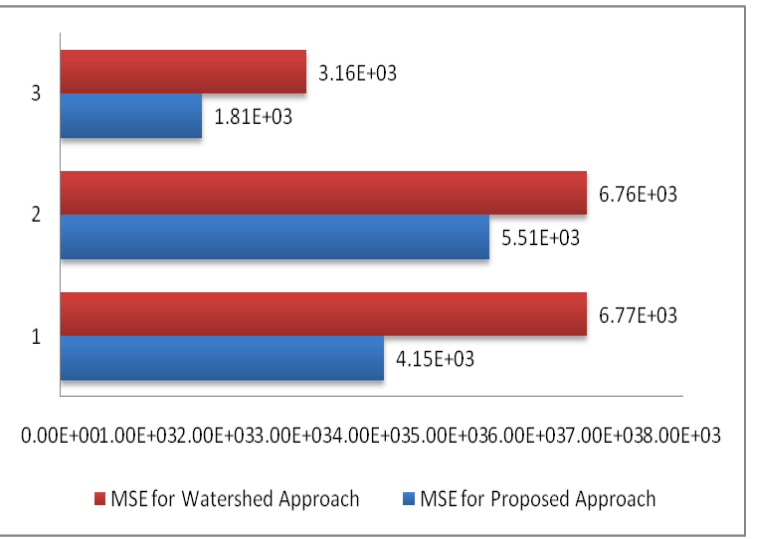

Chart 2.1: MSE Comparison for the Image 2 


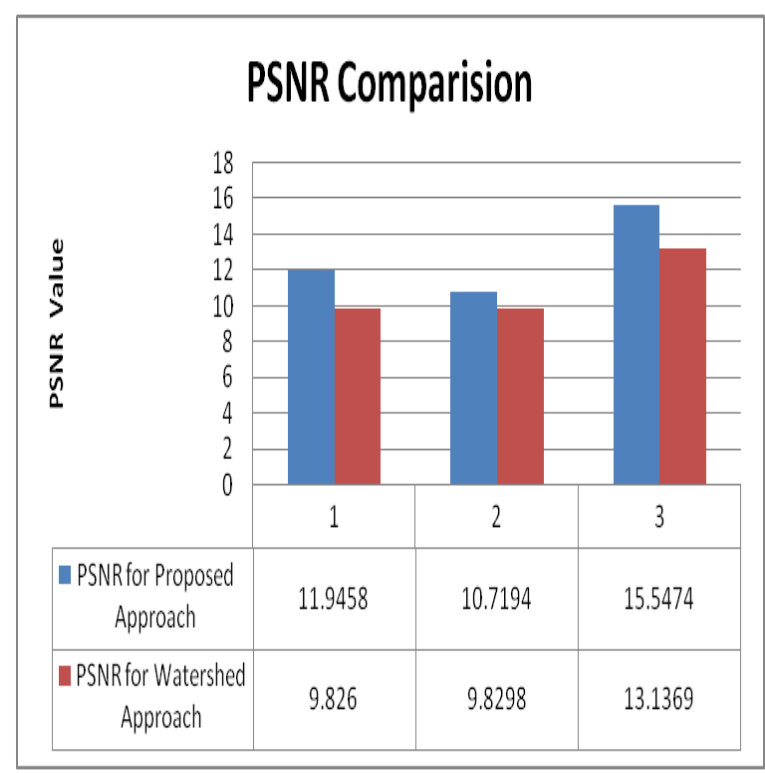

Chart 2.2: PSNR Comparison for the Image 2

From the resultant segmented images (for both image 1 and image 2), it is clearly visible that the proposed approach is free from 'over segmentation' problem that generally occurs in case of watershed approach. Also, the results of the segmentation for both the images have implied low MSE values and high PSNR values in comparison to that of watershed approach. We know that a low MSE value and a high PSNR value always signify good image segmentation. So, the proposed approach has succeeded to produce a better segmentation result.

\section{CONCLUSION \& FUTURE WORK:}

Color image segmentation enhances the image analysis process in a great way. Our human eyes always have a tendency to more accurately identify an object in color form than the same in gray form. So, today's image processing research is giving importance in finding out an efficient and optimal technique for color image segmentation. In this paper, we have proposed an efficient color image segmentation approach. The proposed approach is the combination of two different segmentation techniques: soft clustering and region based techniques. We picked up FCM from the soft clustering techniques and Meyer's Watershed Algorithm from the region based techniques. Also, here we are giving importance on the analysis of color space by considering its impact on the whole segmentation performance. We have chosen LAB color space and extracted L channel for our segmentation task. Normally watershed approaches lead to over segmentation problems. So they require some pre processing tasks to deal with this problem. Here FCM and Sobel filter together handle this pre processing issue. And it works well as the results of the experiments are found free from over segmentation. Also MSE and PSNR values of the proposed approach are quite better than those of the watershed approach. So, our proposed approach can be considered as an effective and optimal color image segmentation approach.

As our future research work, we will try to deal with some post processing techniques that can be added to our proposed approach to enhance its performance. HSV color space is also found improving color image segmentation result. So, as our future work, we will try to analyze the properties of HSV color space with respect to color image segmentation and try to extend our proposed work to this color space.

\section{REFERENCES}

[1] Chris Solomon, Toby Breckon, "Fundamentals of Digital Image Processing", ISBN 9780470844724.

[2] R. M. Haralick, L. G.Shapiro, "Image Segmentation Techniques",CVGIP, vol. 29, 1985,pp. 100-132.

[3] S. M. Aqil Burney, Humera Tariq, “ K-Means Cluster Analysis for Image Segmentation", International Journal of Computer Applications (0975 - 8887) Volume 96-No.4, June 2014, pp. 1-8.

[4] Amanpreet Kaur Bhogal, Neeru Singla,Maninder Kaur, "Color image segmentation using k-means clustering algorithm", International Journal on Emerging Technologies 1(2), 2010,pp. 18-20.

[5] D.J. Bora, A.K. Gupta, "Clustering Approach Towards Image Segmentation: An Analytical Study", IJRCAR,Vol2,Issue 7,July 2014, pp. 115-124

[6] D.J. Bora, A.K. Gupta, F.A. Khan, " Comparing the Performance of $\mathrm{L}^{*} \mathrm{~A} * \mathrm{~B} *$ and HSV Color Spaces with Respect to Color Image Segmentation", International Journal of Emerging Technology and Advanced Engineering, Volume 5, Issue 2, February 2015, pp. 192203.

[7] C Mythili, V.kavitha, "Color Image Segmentation using ERKFCM", International Journal of Computer Applications 41(20), March 2012, pp.21-28.

[8] Juraj Horvath, "Image Segmentation using Fuzzy Cmeans", SAMI 2006.

[9] Indah Soesanti,Adhi Susanto,Thomas Sri Widodo,Maesadji Tjokronagoro, "Optimized Fuzzy Logic Application For MRI Brain Images Segmentation", International Journal of Computer Science \& Information Technology (IJCSIT) Vol 3, No 5, Oct 2011, pp. 137-146.

[10] Le Capitaine H, Frélicot C, "A fast fuzzy c-means algorithm for color image segmentation", In EUSFLAT LFA 2011, Aix-les-Bains, France, 18-22 July 2011. Paris: Atlantis; 2011, pp. 1074-1081.

[11] D.J. Bora, A.K. Gupta, "A Novel Approach Towards Clustering Based Image Segmentation", International Journal of Emerging Science and Engineering (IJESE) ISSN: 2319-6378, Volume-2 Issue-11, September 2014,pp. 6-10.

[12] Lamia Jaafar Belaid, Walid Mourou, "Image Segmentation: A Watershed Transformation Algorithm", Image Anal Stereol, 2009;28, pp.93-102

[13] Rahman, M.H., Islam, M.R., "Segmentation of color image using adaptive thresholding and masking with watershed algorithm," Informatics, Electronics \& Vision (ICIEV), 2013 International Conference on, vol., no., pp.1,6, 17-18 May 2013

[14] Amit Chaudhary , Tarun Gulati, "Segmenting Digital Images Using Edge Detection”, International Journal of Application or Innovation in Engineering \& Management, Volume 2, Issue 5, May 2013, pp. 319-323.

[15] Hunter, RichardSewall (July 1948). "photoelectric colordifference meter". Josa 38 (7): 661. (Proceedings of the winter meeting of the optical society of America) 
[16] Hunter, RichardSewall (December 1948). "Accuracy, precision, and stability of new photo-electric colordifference meter". Josa 38 (12): 1094. (Proceedings of the thirty-third annual meeting of the optical society of America)

[17] CIELAB,

http://dba.med.sc.edu/price/irf/Adobe_tg/models/cielab.h tml

[18] J. C. Bezdek, "Pattern Recognition with Fuzzy Objective Function Algorithms", Plenum Press, New York, 1981.

[19] D.J. Bora, A.K.Gupta, "Impact of Exponent Parameter Value for the Partition Matrix on the Performance of Fuzzy C Means Algorithm", arXiv preprint arXiv:1406.4007

[20] Irwin Sobel, 2014, History and Definition of the Sobel Operator

[21] Raman Maini, Dr. Himanshu Aggarwal, "Study and Comparison of Various Image Edge Detection Techniques, "International Journal of Image Processing (IJIP), Jan-Feb 2009, Volume (3) Issue (1), pp.1-11.

[22] S. A. Salem, N. V. Kalyankar and S. D. Khamitkar, "Image Segmentation By Using Edge Detection",
(IJCSE) International Journal On Computer Science And Engineering, vol. 2, no. 3, 2010,pp. 804-807.

[23] Jos B.T.M. Roerdink ,Arnold Meijster, "The Watershed Transform: Definitions, Algorithms and Parallelization Strategies", Fundamenta Informaticae 41 (2001) 1872281 IOS Press, pp. 1-40.

[24] MATLAB Notes http://www.mathworks.de/company/newsletters/news_ notes/win02/watershed.html

[25] Meyer, Fernand, "Topographic distance and watershed lines," Signal Processing, Vol. 38, July 1994, pp. 113125

[26] MATLAB Demo Images http://www.mathworks.in/products/image

[27] Berkeley Segmentation Dataset: Images http://www.eecs.berkeley.edu/Research/Projects/CS/visio n/bsds/BSDS300/html/dataset/images.html

[28] T. Veldhuizen. "Measures of image quality," 2010 http://homepages.inf.ed.ac.uk/rbf/CVonline/LOCAL_CO PIES/VELDHUIZEN/node18.html 